\title{
Chaos and quantum-classical correspondence via phase-space distribution functions
}

\author{
Jiangbin Gong* and Paul Brumer \\ Chemical Physics Theory Group, Department of Chemistry, University of Toronto, Toronto, Canada M5S 3 H6
}

(Received 25 July 2003; published 4 December 2003)

\begin{abstract}
Quantum-classical correspondence in conservative chaotic Hamiltonian systems is examined using a uniform structure measure for quantal and classical phase-space distribution functions. The similarities and differences between quantum and classical time-evolving distribution functions are exposed by both analytical and numerical means. The quantum-classical correspondence of low-order statistical moments is also studied. The results shed considerable light on quantum-classical correspondence.
\end{abstract}

DOI: 10.1103/PhysRevA.68.062103

PACS number(s): 03.65.Sq, 05.45.Mt

\section{INTRODUCTION}

The quantum dynamics of systems that are classically chaotic has been a subject of considerable interest for nearly three decades. Chaos, usually defined as the exponential sensitivity of phase-space trajectories to slight changes of the initial conditions, resists direct translation to the natural Hilbert space setting of quantum mechanics since there do not exist well-defined trajectories within the conventional interpretation of quantum mechanics [1]. Hence, many studies on quantum chaos in the literature have been dedicated to the relatively modest study of quantum manifestations of classical chaos [2,3]. Actual quantum-classical correspondence (QCC) in classical chaotic systems is still one of the outstanding issues in quantum theory.

Considerations of quantum-classical correspondence that rely upon the standard formulations of quantum and classical mechanics are at a great disadvantage. Specifically, classical mechanics does not admit wave functions and quantum mechanics does not admit trajectories. Hence one is faced with trying to connect two theories which do not even have the same basic rudimentary elements.

A far more enlightening approach is to utilize the Hilbertspace formulation of both quantum [4] and classical [5] mechanics where the central element is the density operator in both formulations. By choosing to deal with the phase-space representation of quantum mechanics one can then compare the classical and quantum dynamics of distributions in phase space. Indeed, all the associated machinery of commutation rules, eigenvalues, eigenstates, etc., can be used to formally analyze the quantum-classical correspondence of both integral and chaotic systems [6].

Recognizing that phase-space distributions allow for a common view of classical and quantum mechanics (see also Refs. [7-10]) suggests that we consider the nature of chaos as it manifests itself in phase-space distributions, as distinct from classical phase-space trajectories. To this end we [11], and others $[12,13]$, have developed a criterion for chaos in terms of the structure of phase-space distribution functions.

In this paper, the distribution function approach to QCC in classically chaotic systems is carefully examined and sub-

\footnotetext{
* Present address: Department of Chemistry and The James Franck Institute, University of Chicago, Chicago, IL 60637, USA.
}

stantially extended. The extension is from one-dimensional chaotic systems in previous work $[11,12]$ to two-dimensional conservative systems, from the special case of uniform dynamical instabilities to the general case of nonuniform stretching and contraction rates fluctuating with time and phase-space location. That is, armed with a measure of the structure of phase-space distribution functions, it becomes possible to quantitatively investigate the similarities and differences between quantal and classical distribution functions in a broad class of smooth, conservative, and strongly chaotic Hamiltonian systems. In particular, this paper displays (1) the rich transient behavior of the dynamics of distribution functions shared by quantum and classical dynamics before a QCC break time $t_{b}$, a quantity which scales with the logarithm of $\hbar$, (2) a simple but enlightening description of the break regime of QCC in the time development of phasespace structure, and (3) interesting QCC in low-order statistical moments during a complex relaxation process with a time scale much larger than $t_{b}$.

We note in passing that the distribution function strategy is also of considerable interest to the fundamental understanding of decoherence in quantum vs classical mechanics. For example, the structure of the classical or quantum distribution functions determines properties of the system when coupled to an environment [14,15]. Hence, QCC from a distribution function viewpoint is useful to the understanding of short-time quantum decoherence rates vs rates of classical entropy production [16]. Furthermore, QCC between quantal and classical distribution functions is closely related to the issue of decoherence in the presence of an environment that has a chaotic classical limit [17].

The model calculations in this paper are based on a strongly chaotic system: the quartic oscillator model [18]. The Hamiltonian is, in dimensionless scaled variables [19], given by

$$
H\left(q_{1}, q_{2}, p_{1}, p_{2}\right)=\frac{p_{1}^{2}}{2}+\frac{p_{2}^{2}}{2}+\frac{\alpha}{2} q_{1}^{2} q_{2}^{2}+\frac{\beta}{4}\left(q_{1}^{4}+q_{2}^{4}\right) .
$$

When $\alpha=\beta$ or $3 \beta$, this system is completely integrable. For very large values of $\alpha / \beta$ the system is strongly chaotic, with the characteristic Lyapunov exponent being an order of magnitude larger than that of other conservative chaotic systems, e.g., the Hénon-Heiles system. 
This paper is organized as follows. Section II briefly reviews the distribution function approach to QCC in chaotic systems. Special emphasis is put on a structure measure of classical and quantal distribution functions, and on our definitions of classical and quantal finite-time Lyapunov exponents. The paper then proceeds to present results in order of increasing time scales. In Sec. III, QCC is studied for initially positive-definite Wigner functions at early times. In Sec. IV, a simple analytical understanding of the break time regime of QCC is provided, followed by supporting numerical results. We then consider, in Sec. V, QCC in some loworder statistical moments for much larger time scales. A summary is given in Sec. VI.

\section{LYAPUNOV EXPONENTS IN DISTRIBUTION FUNCTION DYNAMICS}

\section{A. Classical distribution function dynamics}

Classical chaos is usually defined as the extreme sensitivity of trajectories to slight changes in initial conditions. Quantitatively, it is described by a positive maximum Lyapunov exponent or by the Kolomogorov entropy. Consider a conservative Hamiltonian system with two degrees of freedom with dimensionless canonical variables $q_{1}, q_{2}, p_{1}, p_{2}$. A phase-space point is characterized by a fourdimensional column vector $\boldsymbol{\gamma} \equiv\left(q_{1}, q_{2}, p_{1}, p_{2}\right)^{\dagger}$. For brevity we introduce the antisymmetric matrix $\mathbf{J}=\left(\begin{array}{cc}\mathbf{0} & \mathbf{1} \\ -\mathbf{1} & 0\end{array}\right)$, where $\mathbf{0}$ and 1 are $2 \times 2$ zero and unit matrices, respectively. The canonical equations of motion (i.e., Hamilton's equations) then read as $\dot{\gamma}=\mathbf{J} \partial H / \partial \gamma$. The sensitivity of classical trajectories to initial conditions is described by the stability matrix $\mathbf{M}_{i j}[\gamma(0), t] \equiv \partial \gamma_{i}(t) / \partial \gamma_{j}(0)$, and the maximal Lyapunov exponent $\lambda$ is given by

$$
\lim _{t \rightarrow \infty} \frac{1}{t} \ln \{|\mathbf{M}[\gamma(0), t] \eta|\}=\lambda[\gamma(0)]
$$

where $\eta$ is a vector in the tangent space. The stability matrix $\mathbf{M}$ is symplectic and its time evolution is governed by the differential equation

$$
\dot{\mathbf{M}}=\mathbf{J} \frac{\partial^{2} H}{\partial \gamma^{2}} \mathbf{M}
$$

with

$$
\mathbf{M}(0)=\mathbf{1}
$$

A simple application of Liouville's theorem leads to an alternative expression for the maximal Lyapunov exponent in terms of classical distribution function dynamics $[12,11]$. Let $\rho_{t}$ denote a well-behaved classical probability distribution and $\xi$ denote an arbitrary infinitesimal vector in phase space. Then, from the incompressibility of the Liouville density function, one has

$$
\rho_{t}[\gamma(t)]+\nabla \rho_{t}[\gamma(t)] \mathbf{M} \xi=\rho_{0}[\gamma(0)]+\nabla \rho_{0}[\gamma(0)] \xi .
$$

Further, using $\mathbf{J}^{2}=-1$ and $\mathbf{J} \mathbf{M}^{\dagger} \mathbf{J}=-\mathbf{M}^{-1}$, one obtains

$$
\mathbf{M J} \boldsymbol{\nabla} \rho_{0}[\gamma(0)]=\mathbf{J} \boldsymbol{\nabla} \rho_{t}[\gamma(t)] .
$$

Substituting Eq. (6) into Eq. (2) yields

$$
\begin{aligned}
\lambda[\gamma(0), t] & =\lim _{t \rightarrow \infty} \frac{1}{t} \ln \left|\mathbf{J} \boldsymbol{\nabla} \rho_{t}[\gamma(t)]\right| \\
& =\lim _{t \rightarrow \infty} \frac{1}{t} \ln \left|\boldsymbol{\nabla} \rho_{t}[\gamma(t)]\right| .
\end{aligned}
$$

Note that Eq. (7) defines the Lyapunov exponent in terms of phase-space distribution properties. Specifically, the faster the structure of $\rho_{t}$ increases, the larger $\lambda$ is.

In accord with Ref. [11], we define a particular ensemble average of the gradients of distribution functions as $\chi_{2 c}$, i.e.,

$$
\begin{aligned}
\chi_{2 c}(t) & \equiv\left[\frac{\int\left|\boldsymbol{\nabla} \rho_{t}(\gamma)\right|^{2} d \gamma}{\int \rho_{t}^{2}(\gamma) d \gamma}\right]^{1 / 2} \\
& =\left[-\frac{\int \rho_{t}(\gamma) \nabla^{2} \rho_{t}(\gamma) d \gamma}{\int \rho_{t}^{2}(\gamma) d \gamma}\right]^{1 / 2} .
\end{aligned}
$$

Using Eqs. (6) and (8), we have

$$
\chi_{2 c}(t)=\left[\frac{1}{\int \rho_{0}^{2}(\gamma) d \gamma} \int\left|\mathbf{J M}(\gamma, t) \mathbf{J} \boldsymbol{\nabla} \rho_{0}(\gamma)\right|^{2} d \gamma\right]^{1 / 2}
$$

The physical significance of $\chi_{2 c}(t)$ becomes apparent when one considers the Fourier transform of the distribution function. That is, suppose $\rho_{t}(\gamma)$ $=\left[1 /(2 \pi)^{4}\right] \int d \mathbf{k} \exp (2 \pi i \mathbf{k} \cdot \gamma) \rho_{t}(\mathbf{k})$, where $\rho_{t}(\mathbf{k})$ denotes the Fourier component evaluated at the 4-dimensional wave vector $\mathbf{k}$. Then

$$
\chi_{2 c}^{2}(t)=\frac{\int d \mathbf{k} \mathbf{k}^{2}\left|\rho_{t}(\mathbf{k})\right|^{2}}{\int d \mathbf{k}\left|\rho_{t}(\mathbf{k})\right|^{2}},
$$

showing that $\chi_{2 c}$ is the root-mean-square radius of the Fourier transform of the distribution function, and thus serves as a measure of classical phase-space structure. That is, the larger $\chi_{2 c}$ is, the more structured $\rho_{t}(\gamma)$ is.

For completely integrable dynamics there exists a special set of generalized coordinates: action variables $I_{1}, I_{2}$ and angle variables $\theta_{1}, \theta_{2}$. In this representation, the Hamiltonian depends only on the action variables that are constants of motion. For such cases, Eq. (3) has the simple solution

$$
\mathbf{M}=1+\mathbf{J} \frac{\partial^{2} H}{\partial \gamma^{2}} t
$$


Substituting this explicit time dependence of the stability matrix into Eq. (9) gives the following long-time behavior of $\chi_{2 c}$ :

$$
\lim _{t \rightarrow \infty} \frac{\chi_{2 c}(t)}{t}=\left[\frac{1}{\int \rho_{0}^{2}(\gamma) d \gamma} \int\left|\frac{\partial^{2} H}{\partial \gamma^{2}} \mathbf{J} \nabla \rho_{0}(\gamma)\right|^{2} d \gamma\right]^{1 / 2} .
$$

Hence, for regular dynamics the structure of distribution functions, as measured by $\chi_{2 c}$, asymptotically shows a linear time dependence in the action-angle representation. However, $\chi_{2 c}(t)$ may show a polynomial time dependence in other canonical representations.

By contrast, for chaotic dynamics it was shown that [11]

$$
\lim _{t \rightarrow \infty} \frac{1}{t} \ln \chi_{2 c}(t)=\lambda_{2},
$$

where $\lambda_{2}$ is the so-called second-order generalized maximal Lyapunov exponent. That is, in the chaotic case the rootmean-square Fourier radius of distribution functions increases, asymptotically, at an exponential rate of $\lambda_{2}$. Since a given resolution limit $\delta$ corresponds to the inability to account for Fourier modes larger than $1 / \delta$, chaos can be understood as a kind of exponential loss of accuracy, or of information, encoded in the Fourier basis expansion of the initial distribution function.

By definition, the (generalized) Lyapunov exponent $\lambda_{2}$ is an asymptotic property, relevant as time goes to infinity. Realistically, however, it is finite-time properties of classical chaotic dynamics that are of real interest to the study of QCC. To this end it is useful to introduce a finite-time Lyapunov exponent. Based upon Eq. (13), we define the finite-time Lyapunov exponent in terms of the average exponential increase rate of $\chi_{2 c}(t)$ over time $t$ :

$$
\lambda_{2 c}(t) \equiv \frac{1}{t} \ln \left[\frac{\chi_{2 c}(t)}{\chi_{2 c}(0)}\right],
$$

with

$$
\lim _{t \rightarrow+\infty} \lambda_{2 c}(t)=\lambda_{2}
$$

We examine this quantity over various time scales below.

\section{B. Quantal analog of classical finite-time Lyapunov exponents}

From the ensemble point of view, QCC is best understood by comparing the classical Liouville equation with the quantum von Neumann equation in a phase-space representation, e.g., the Wigner-Weyl representation. Specifically, given Eq. (9), which provides a quantitative diagnostic for characterizing classical chaos using classical distribution functions, it becomes straightforward to define the quantum analog of classical Lyapunov exponents using quantal distribution functions. By analogy with Eq. (8) we define the measure $\chi_{2 q}$ for quantal phase-space structure as

$$
\chi_{2 q} \equiv\left[\frac{\int\left|\nabla \rho^{W}(\gamma)\right|^{2} d \gamma}{\int\left(\rho^{W}\right)^{2}(\gamma) d \gamma}\right]^{1 / 2}
$$

where $\rho^{W}(\gamma)$ is the Wigner function of a quantum state. Accordingly, in the Fourier space of quantal distribution function,

$$
\chi_{2 q}^{2}=\frac{\int d \mathbf{k k}^{2}\left|\rho_{t}^{W}(\mathbf{k})\right|^{2}}{\int d \mathbf{k}\left|\rho_{t}^{W}(\mathbf{k})\right|^{2}}
$$

where $\rho_{t}^{W}(\gamma)=\left[1 /(2 \pi)^{4}\right] \int d \mathbf{k} \exp (2 \pi i \mathbf{k} \cdot \gamma) \rho_{t}^{W}(\mathbf{k})$. Hence, $\chi_{2 q}$ is the root-mean-square Fourier radius of the Wigner function. Further, finite-time Lyapunov exponents $\lambda_{2 q}(t)$ for quantum distribution function dynamics can be defined by direct analogy to $\lambda_{2 c}(t)$, i.e.,

$$
\lambda_{2 q}(t) \equiv \frac{1}{t} \ln \left[\frac{\chi_{2 q}(t)}{\chi_{2 q}(0)}\right] .
$$

Interestingly, $\chi_{2 q}$ has an equivalent expression that is easier to handle. Suppose $\hat{\rho}$ is the density-matrix operator associated with the Wigner function $\rho^{W}$ and $\hat{\gamma}_{i}$ is the operator associated with the classical canonical variable $\gamma_{i}$ (e.g., $\left.q_{i}, p_{i}\right)$. A simple calculation $[11,12]$ then shows that

$$
\chi_{2 q}^{2}=2 \sum_{i} \frac{\operatorname{Tr}\left(\hat{\rho}^{2} \hat{\gamma}_{i}^{2}-\hat{\rho} \hat{\gamma}_{i} \hat{\rho} \hat{\gamma}_{i}\right)}{\hbar^{2} \operatorname{Tr}\left(\hat{\rho}^{2}\right)}
$$

Of particular interest is the pure state case, in which $\hat{\rho}^{2}=\hat{\rho}$ and, as a result,

$$
\chi_{2 q}^{2}=\frac{2}{\hbar^{2}} \sum_{i}\left(\left\langle\hat{\gamma}_{i}^{2}\right\rangle-\left\langle\hat{\gamma}_{i}\right\rangle^{2}\right),
$$

where $\langle\cdots\rangle$ represents ensemble expectation values. As shown below, the analogous classical expression is far more complex.

Consider then the quantum counterpart of Eq. (15). Since Eq. (19) indicates that $\chi_{2 q}^{2} \leqslant 2 \Sigma_{i} \operatorname{Tr}\left(\hat{\rho}^{2} \widehat{\gamma}_{i}^{2}\right) / \hbar^{2} \operatorname{Tr} \hat{\rho}^{2}$, we have that $\chi_{2 q}$ has an upper bound for any bounded Hamiltonian system. As such, for fixed $\hbar$ and bounded systems,

$$
\lim _{t \rightarrow+\infty} \lambda_{2 q}(t)=0
$$

This reproduces the widely accepted result that bounded quantum systems cannot exhibit chaos in the strict sense.

However, as noted above, what is of interest to the study of QCC are the transient properties of $\lambda_{2 q}(t)$ vs $\lambda_{2 c}(t)$. Consider, for example, a two-degree-of-freedom system $H$ $=H\left(q_{1}, q_{2}, p_{1}, p_{2}\right)$. The quantum von Neumann equation in terms of the Wigner function $\rho^{W}$ is given by 


$$
\begin{aligned}
\frac{\partial \rho^{W}}{\partial t}= & \left\{H, \rho^{W}\right\}+\sum_{\left(l_{1}+l_{2}\right)>1, \text { odd }} \frac{(\hbar / 2 i)^{\left(l_{1}+l_{2}-1\right)}}{l_{1} ! l_{2} !} \\
& \times \frac{\partial^{\left(l_{1}+l_{2}\right)} V\left(q_{1}, q_{2}\right)}{\partial q_{1}^{l_{1}} \partial q_{2}^{l_{2}}} \frac{\partial^{\left(l_{1}+l_{2}\right)} \rho^{W}}{\partial p_{1}^{l_{1}} \partial p_{2}^{l_{2}}}
\end{aligned}
$$

where the first term on the right-hand side is the classical Poisson bracket and the second term represents the sum over an infinite series of "quantum corrections." Consider the short-time limit of $\lambda_{2 q}(t)$. Using Eqs. (16) and (22) we have

$$
\begin{aligned}
\lambda_{2 q}(0)= & \frac{1}{\int\left|\boldsymbol{\nabla} \rho_{0}^{W}\right|^{2} d \gamma}\left[\int\left(\boldsymbol{\nabla} \rho_{0}^{W}\right)^{T}\left(\frac{\partial^{2} H}{\partial \gamma^{2}}\right) \mathbf{J}\left(\boldsymbol{\nabla} \rho_{0}^{W}\right) d \gamma\right. \\
& +\int\left(\boldsymbol{\nabla} \rho_{0}^{W}\right)^{T} d \gamma \sum_{\left(l_{1}+l_{2}\right)>1, o d d} \frac{(\hbar / 2 i)^{\left(l_{1}+l_{2}-1\right)}}{l_{1} ! l_{2} !} \\
& \left.\times \nabla \frac{\partial^{\left(l_{1}+l_{2}\right)} V\left(q_{1}, q_{2}\right)}{\partial q_{1}^{l_{1}} \partial q_{2}^{l_{2}}} \frac{\partial^{\left(l_{1}+l_{2}\right)} \rho_{0}^{W}}{\partial p_{1}^{l_{1}} \partial p_{2}^{l_{2}}}\right],
\end{aligned}
$$

where, obviously, the first term on the right-hand side of Eq. (23) corresponds to the contribution from the classical Poisson bracket and all other terms represent quantum corrections.

Some aspects of the distribution function strategy outlined above have been applied to the Arnold-cat map model $[11,12]$, where the stretching and contraction mechanism is uniform over the entire phase space. However, rich transient behavior of finite-time Lyapunov exponents, as implied in our derivation of the explicit state dependence of $\lambda_{2 q}(0)$, has not been explored thus far. The following section is devoted to both analytical and numerical studies on this subject.

\section{SHORT-TIME CORRESPONDENCE}

To examine the QCC in short-time dynamics it is useful to consider the classical analog of $\lambda_{2 q}(0)$, i.e., $\lambda_{2 c}(0)$ as the extreme short-time limit of classical finite-time Lyapunov exponents. Using Eqs. (3), (4), and (9), we have

$$
\lim _{t \rightarrow 0} \frac{d \chi_{2 c}^{2}}{d t}=\frac{2}{\int \rho_{0}^{2}(\gamma) d \gamma} \int\left(\nabla \rho_{0}\right)^{T}\left(\frac{\partial^{2} H}{\partial \gamma^{2}}\right) \mathbf{J}\left(\nabla \rho_{0}\right) d \gamma
$$

Substituting Eq. (24) into Eq. (14) gives the zero-time limit of $\lambda_{2 c}(t)$,

$$
\lambda_{2 c}(0)=\frac{\int\left(\boldsymbol{\nabla} \rho_{0}\right)^{T}\left(\frac{\partial^{2} H}{\partial \gamma^{2}}\right) \mathbf{J}\left(\nabla \rho_{0}\right) d \gamma}{\int\left|\nabla \rho_{0}\right|^{2} d \gamma}
$$

Here, $\lambda_{2 c}(0)$ is seen to be the average of $\partial^{2} H / \partial \gamma^{2}$ weighted by gradients of the initial distribution function. Not surprisingly, Eq. (25) resembles the first term on the right-hand side of Eq. (23). As $\lambda_{2 c}(0)$ reflects an ensemble average of instantaneous density fluctuations, it depends strongly on the shape and location of the initial classical distribution function. Hence, as in the quantum case, there exists very rich transient behavior in the time development of phase-space structure, an interesting feature that has often been ignored in previous QCC studies.

To consider quantum effects induced solely by the dynamics suggests that we choose an initial quantal distribution function that is as classical as possible so that differences between classical and quantum dynamics evidently arise from the dynamics. This suggests that the initial Wigner function should be chosen as positive definite, so that it can be interpreted as a classical probability distribution. It is well known that for one-dimensional pure state dynamics the only positive-definite Wigner function is the Gaussian distribution function [20,21], which takes the following general form:

$$
\begin{aligned}
\rho_{r, \eta, \bar{q}, \bar{p}}^{W}= & \frac{1}{\pi \hbar} \exp \left[-\frac{2 \eta^{2}}{\hbar^{2}}(p-\bar{p})^{2}-\frac{(q-\bar{q})^{2}}{2 \eta^{2}\left(1-r^{2}\right)}\right. \\
& \left.+\frac{2 r}{\hbar\left(1-r^{2}\right)^{1 / 2}}(q-\bar{q})(p-\bar{p})\right]
\end{aligned}
$$

where $r, \eta, \bar{q}, \bar{p}$ are parameters and where

$$
\begin{gathered}
\langle q\rangle=\bar{q}, \quad\langle p\rangle=\bar{p},\left\langle q^{2}-\bar{q}^{2}\right\rangle=\eta^{2} \\
\left\langle p^{2}-\bar{p}^{2}\right\rangle=\frac{\hbar^{2}}{4 \eta^{2}\left(1-r^{2}\right)}, \quad\langle p q\rangle-\langle p\rangle\langle q\rangle=\frac{\hbar r}{2\left(1-r^{2}\right)^{1 / 2}} .
\end{gathered}
$$

This Gaussian form corresponds to the so-called correlated coherent states [22], whose coordinate representation is given by

$$
\begin{aligned}
\Psi(q)= & \frac{1}{\left(2 \pi \eta^{2}\right)^{1 / 4}} \exp \left[-\frac{q^{2}}{4 \eta^{2}}\left(1-\frac{i r}{\left(1-r^{2}\right)^{1 / 2}}\right)+\frac{\alpha q}{\eta}\right. \\
& \left.-\frac{1}{2}\left(\alpha^{2}+|\alpha|^{2}\right)\right]
\end{aligned}
$$

where $\alpha$ is a complex constant given by $\bar{q} /(2 \eta)+i[\bar{p} \eta / \hbar$ $\left.-\bar{q} r /\left(2 \eta \sqrt{1-r^{2}}\right)\right]$. In particular, for the case of $r=0,\left\langle q^{2}\right.$ $\left.-\bar{q}^{2}\right\rangle\left\langle p^{2}-\bar{p}^{2}\right\rangle=\hbar^{2} / 4$, corresponding to the minimumuncertainty-product state, i.e., the coherent state.

For the two-degree-of-freedom system examined below, one would choose two-dimensional Gaussian states. The initial quantal distribution function $\rho_{0}^{W}$ and classical distribution function $\rho_{0}$ are thus chosen as the following:

$$
\rho_{0}^{W}=\rho_{0}=\rho_{r_{1}, \eta_{1}, \bar{q}_{1}, \bar{p}_{1}}^{W} \otimes \rho_{r_{2}, \eta_{2}, \bar{q}_{2}, \bar{p}_{2}}^{W} .
$$

Substituting this initial state into Eq. (25) and approximating the average of the derivatives of $V\left(q_{1}, q_{2}\right)$ as the derivative evaluated at the centroid of the Gaussian distribution, denoted $\partial^{2} V\left(\bar{q}_{1}, \bar{q}_{2}\right) / \partial \bar{q}_{i}^{2}$, one gets 


$$
\begin{aligned}
\lambda_{2 c}(0)= & \frac{1}{\sum_{i=1,2}\left[2 \eta_{i}^{2}+\frac{\hbar^{2}}{2\left(1-r_{i}^{2}\right) \eta_{i}^{2}}\right]} \\
& \times \sum_{i=1,2}\left[\frac{\hbar r_{i}}{\left(1-r_{i}^{2}\right)^{1 / 2}}\left(1-\frac{\partial^{2} V\left(\bar{q}_{1}, \bar{q}_{2}\right)}{\partial \bar{q}_{i}^{2}}\right)\right] .
\end{aligned}
$$

It is seen that $\lambda_{2 c}(0)$ depends strongly on both the shape parameters $r_{1}, r_{2}$ and the phase-space locations of the initial distribution function. Two particular situations are worthy noting. First, for the minimum-uncertainty-product state $\left(r_{1}\right.$ $=r_{2}=0$ ), i.e., the two-dimensional coherent state that is commonly used as initial states in QCC studies, Eq. (30) gives $\lambda_{2 c}(0)=0$. Second, when $r_{i}\left[1-\partial^{2} V\left(\bar{q}_{1}, \bar{q}_{2}\right) / \partial \bar{q}_{i}^{2}\right]$ $<0$ for either $i=1$ or $i=2, \lambda_{2 c}(0)$ can be negative. That is, for appropriate shape parameters $r_{1}, r_{2}$ and central coordinates $\bar{q}_{1}, \bar{q}_{2}$, the contraction mechanism associated with chaotic dynamics may initially dominate over the stretching mechanism, giving rise to a reduction in phase-space structure.

The quantum analog of this transient behavior can be examined by considering $\lambda_{2 q}(t)$ in a similar fashion. Specifically, substituting the initial positive-definite Wigner function (29) into Eq. (23), keeping the leading-order quantum correction term in the quantum Liouville equation, one obtains

$$
\begin{aligned}
\lambda_{2 q}(0)= & \lambda_{2 c}(0)-\frac{1}{16 \pi \int\left|\nabla \rho_{0}^{W}\right|^{2} d \gamma} \frac{\partial^{4} V}{\partial \bar{q}_{1}^{2} \partial \bar{q}_{2}^{2}} \\
& \times\left[\frac{r_{2}}{\left(1-r_{2}^{2}\right)^{1 / 2}} \int d q_{1} d p_{1}\left|\nabla \rho_{r_{1}, \eta_{1}, \bar{q}_{1}, \bar{p}_{1}}^{W}\right|^{2}\right. \\
& \left.+\frac{r_{1}}{\left(1-r_{1}^{2}\right)^{1 / 2}} \int d q_{2} d p_{2}\left|\nabla \rho_{r_{2}, \eta_{2}, \bar{q}_{2}, \bar{p}_{2}}^{W}\right|^{2}\right] .
\end{aligned}
$$

Evidently, initial states with $r_{1}=r_{2}=0$ give $\lambda_{2 c}(0)$ $=\lambda_{2 q}(0)=0$. Thus, in this sense, the coherent state is the most classical state of the correlated coherent states; other types of initial states have a leading-order quantum effect proportional to $\partial^{4} V / \partial \bar{q}_{1}^{2} \partial \bar{q}_{2}^{2}$. After carrying out the integrals in Eq. (31) for $\rho_{r, \eta, \bar{q}, \bar{p}}^{W}$ given by Eq. (26), one sees that $\left[\lambda_{2 c}(0)-\lambda_{2 q}(0)\right]$ is proportional to the first power of $\hbar$. Therefore, for relatively large $\hbar$, depending upon the sign of $r_{1}$ and $r_{2}, \lambda_{2 q}(t)$ can be significantly larger or smaller than $\lambda_{2 c}(t)$ at early times. In addition, if there is no quartic term in the potential, i.e., $\partial^{4} V / \partial \bar{q}_{1}^{2} \partial \bar{q}_{2}^{2}=0$ (e.g., in the HénonHeiles model), Eq. (31) shows that $\left[\lambda_{2 c}(0)-\lambda_{2 q}(0)\right]$ is given by smaller terms that are proportional to higher powers of $\hbar$.

Thus far we have only examined correspondence between $\lambda_{2 c}(t)$ and $\lambda_{2 q}(t)$ at $t=0$. For nonzero times, one can utilize numerical methods to compare these quantities. For example,
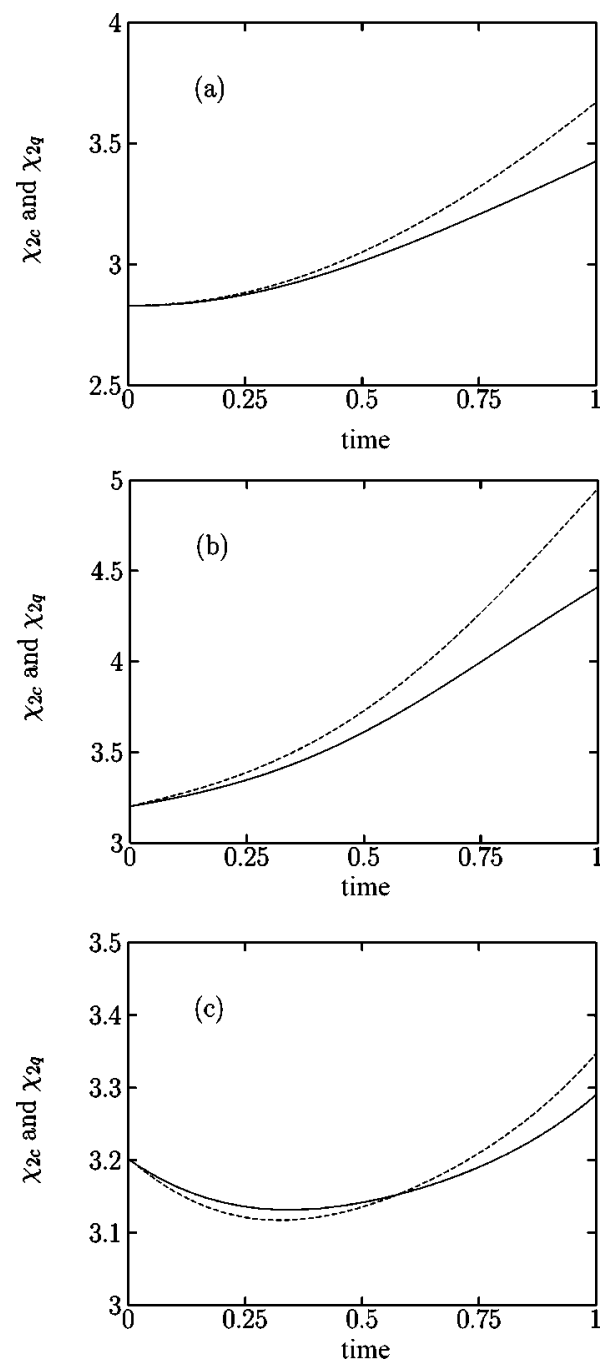

FIG. 1. Time dependence of $\chi_{2 c}(t)$ (dashed line) and $\chi_{2 q}(t)$ (solid line) for three initial Gaussian distributions $(\hbar=0.5)$. The three panels correspond to (a) $r_{1}=r_{2}=0$, (b) $r_{1}=r_{2}=0.6$, (c) $r_{1}$ $=r_{2}=-0.6$. Note that the initial slope of these solid and dashed lines is zero, positive, and negative in (a), (b), and (c), respectively. All variables are in dimensionless units.

consider a coupled quartic oscillator system given by Eq. (1) with $\alpha=1.0$ and $\beta=0.01$, and for $\hbar=0.5,0.05$, and 0.005 . Classical calculations are done by Monte Carlo methods based on Eq. (9), and quantum calculations use the fast Fourier-transform split operator technique [23]. For each case we examine three sets of initial distributions, i.e., (a) $r_{1}=r_{2}=0$, (b) $r_{1}=r_{2}=0.6$, and (c) $r_{1}=r_{2}=-0.6$. For all three cases $\eta_{1}=\eta_{2}=\sqrt{\hbar / 2}$, and the centroid of the initial state is fixed at $\bar{q}_{1}=0.40, \bar{q}_{2}=0.60, \bar{p}_{1}=0.50$, and $\bar{p}_{2}$ $=0.414$. Note that this initial location of the Gaussian distribution gives $\left(1-\partial^{2} V / \partial \bar{q}_{1}^{2}\right)>0$ and $\left(1-\partial^{2} V / \partial \bar{q}_{2}^{2}\right)>0$, a fact that is relevant to the discussions below.

Figures 1-3 display comparisons between $\chi_{2 c}(t)$ and $\chi_{2 q}(t)$. Consider Fig. 1 for the case of $\hbar=0.5$. Here $\partial^{4} V / \partial \bar{q}_{1}^{2} \partial \bar{q}_{2}^{2}=2.0$, and one expects that $\lambda_{2 q}(0)$ can deviate considerably from $\lambda_{2 c}(0)$ for nonzero $r_{1}$ and $r_{2}$. Further, the expectation [from Eq. (31)] is that the initial quantum cor- 

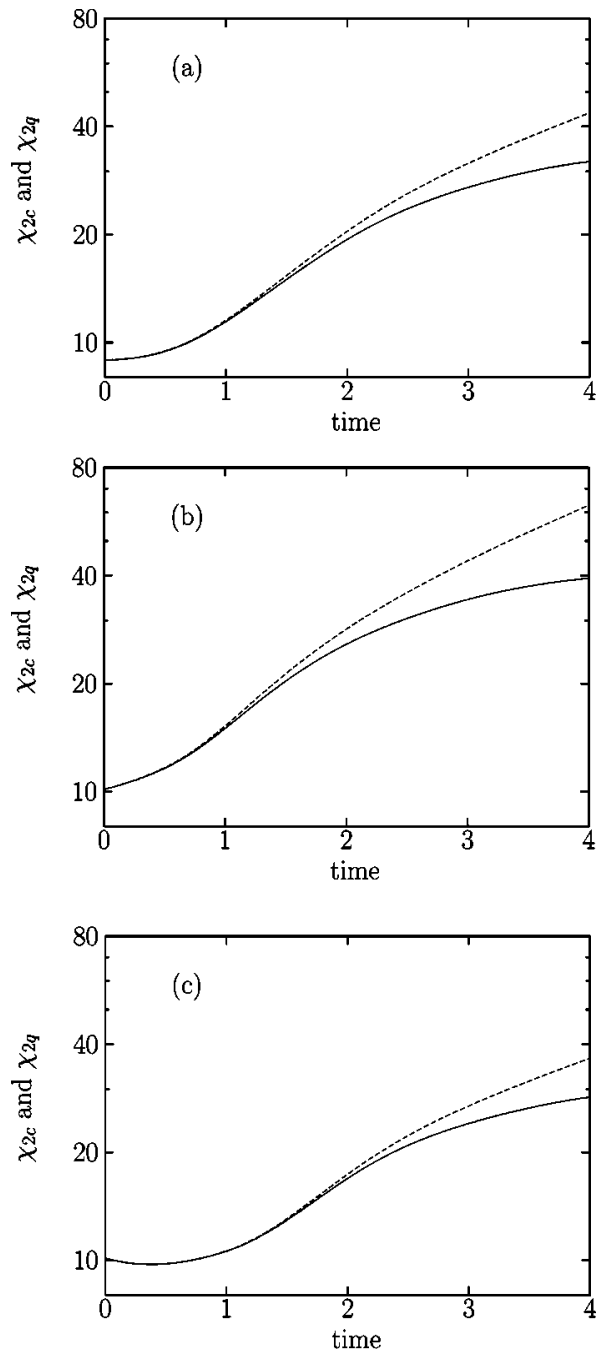

FIG. 2. Same as Fig. 1 except $\hbar=0.05$ and that both $\chi_{2 c}(t)$ and $\chi_{2 q}(t)$ are plotted in the logarithmic scale. The average slope of the curves (up to a certain time) is indicative of the finite-time Lyapunov exponents $\lambda_{2 c}(t)$ and $\lambda_{2 q}(t)$. The break regime of QCC is clearly between $t=1.0$ and $t=1.5$. All variables are in dimensionless units.

rection should be positive when $r_{1}, r_{2}$ are negative and negative when $r_{1}, r_{2}$ are positive. All these predictions are confirmed nicely in our computations. In particular, (i) in Fig. 1(a) $\left(r_{1}=r_{2}=0\right)$, both $\chi_{2 c}(t)$ and $\chi_{2 q}(t)$ assume an initial zero slope; (ii) in Fig. 1(b) $\left(r_{1}=r_{2}=0.6\right)$, the positive slope of $\chi_{2 q}(t)$ at $t=0$ is seen to be smaller than that of $\chi_{2 c}(t)$; and (iii) in Fig. 1(c) $\left(r_{1}=r_{2}=-0.6\right)$, it is indeed seen that at very short times $\chi_{2 q}(t)>\chi_{2 c}(t)$, both of which have negative initial slopes. For all three situations, the quantum effects are so large that QCC is essentially lost at very short times.

Figure 2 shows the corresponding results (with different abscissa scale) with the value of $\hbar$ decreased by a factor of 10. This case is different from that in Fig. 1 insofar as excellent QCC is seen for short times for all three initial conditions. In particular, in Fig. 2(b) the time dependence of both $\chi_{2 c}(t)$ and $\chi_{2 q}(t)$ is seen to be very close to exponential for $t \leqslant 1.5$. However, this is partially because the initial
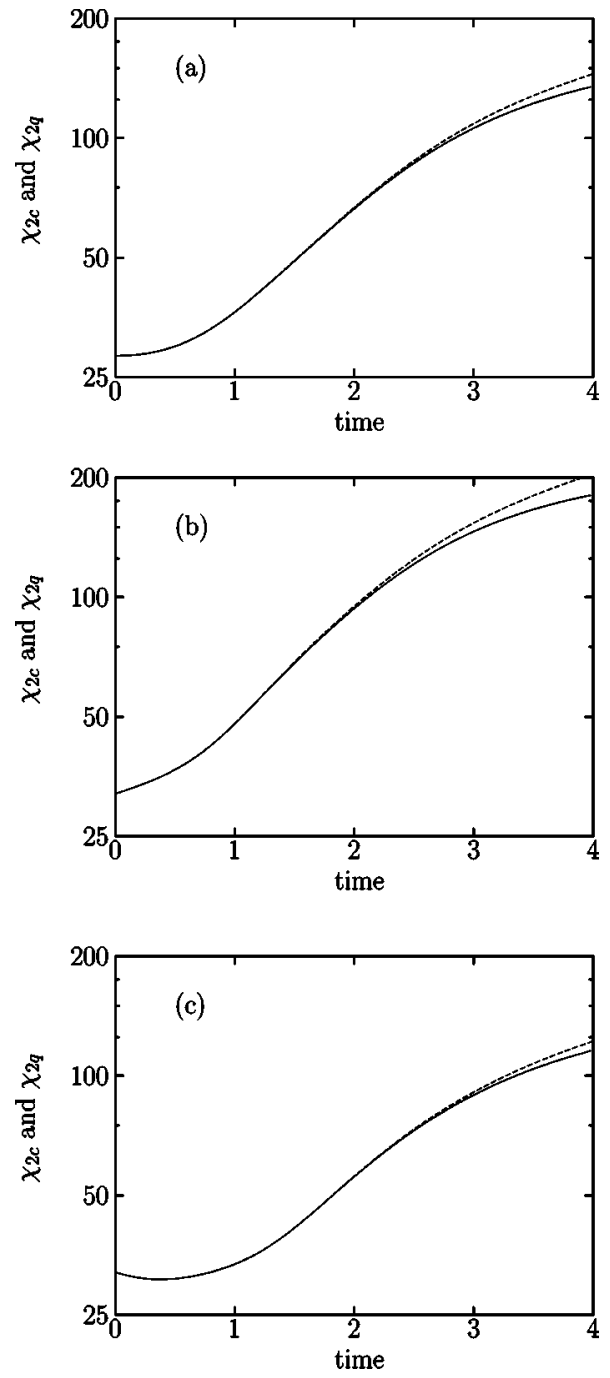

FIG. 3. Same as Fig. 2 except $\hbar=0.005$. The break regime of QCC is clearly between $t=2.0$ and $t=2.5$. For $0.5<t<2.5$ and for all three initial conditions in (a), (b), and (c), there is an exponential increase of phase-space structure for both classical and quantum dynamics. All variables are in dimensionless units.

slope of the curves [i.e., $\lambda_{2 q}(0)$ and $\left.\lambda_{2 c}(0)\right]$ in Fig. 2(b) both happen to be close to the average slope (i.e., finite-time Lyapunov exponents) for longer times (e.g., $t \sim 4$ ). In Fig. 2(c) the transient behavior of the finite-time Lyapunov exponents assumes a completely different nature, i.e., the increase of both $\chi_{2 c}(t)$ and $\chi_{2 q}(t)$ is significantly suppressed for times up to $t=0.5$. Variations in the finite-time Lyapunov exponents can be further seen by comparing $\chi_{2 c}(t=4.0)$ and $\chi_{2 q}(t=4.0)$ in Fig. 2(b) to those in Figs. 2(a) and 2(c): they can differ by a factor as large as 1.5. Note also that Fig. 2 shows that the break regime of QCC (i.e., the time when classical and quantum dynamics no longer agree) is between $t=1.0$ and $t=1.5$, which is of the order of one average period of motion. With increasing time, the agreement between $\chi_{2 q}(t)$ and $\chi_{2 c}(t)$ in Fig. 2 worsens: the classical phasespace structure is seen to increase exponentially on the average, whereas there is no clear sign of a similar exponential increase in the quantum distribution dynamics. 
Besides, we note that since the structure of quantal distribution functions may determine the early-time decoherence rate if the quantum system is open [14], these variations in finite-time Lyapunov exponents imply that even in classically strongly chaotic systems it is still possible to pick out some initial states which are relatively robust to decoherence effects.

As shown in Fig. 3, with a further large decrease of $\hbar$, the break regime of QCC is considerably shifted to $t=2.0-3.0$. The transient behavior caused by different initial states still persists for $t \leqslant 0.5$. After the transient period and before the break time, all quantal distribution functions in Figs. 3(a)(c) emanating from the three different initial conditions are seen to undergo an exponential increase of structure on the average, in excellent agreement with the behavior of classical distribution functions.

\section{THE BREAK REGIME FOR CORRESPONDENCE}

Understanding the QCC break regime is a central problem in the study of correspondence. From the viewpoint adopted here, i.e., via phase-space distribution functions, the break regime is when the classical distribution function begins to develop significantly different structure from the quantal distribution function, i.e., $\chi_{2 c}(t)$ begins to deviate appreciably from $\chi_{2 q}(t)$.

It is tempting to try to use the quantum Liouville equation [Eq. (22)] to study the breakdown of QCC with time. However, this is not an easy task, since, for nonzero times, one cannot analytically deal with distribution function dynamics. Rather, we present a simple description of the QCC break regime by first reinterpreting $\chi_{2 c}^{2}(t)$ and then comparing it with $\chi_{2 q}^{2}(t)$. As will be quite clear, our description also relates the distribution function strategy to the trajectory viewpoint of chaos.

It is convenient to restrict the discussion to the case of the minimum-uncertainty-product state, although the following considerations can be readily extended to the most general case. Further, we focus on pure state dynamics because mixed state dynamics simply makes the study of QCC more complicated, without adding new physical insight.

We begin with the Taylor-series expansion of the stability matrix $\mathbf{M}[\gamma(0), t]$ around $\bar{\gamma}(0)$; i.e.,

$$
\begin{aligned}
\mathbf{M}_{k l}[\gamma(0), t]= & \mathbf{M}_{k l}[\bar{\gamma}(0), t]+\sum_{m} \delta \gamma_{m}(0) \frac{\partial \mathbf{M}_{k l}[\bar{\gamma}(0), t]}{\partial \bar{\gamma}_{m}(0)} \\
& +\frac{1}{2} \sum_{m n} \delta \gamma_{m}(0) \delta \gamma_{n}(0) \frac{\partial^{2} \mathbf{M}_{k l}[\bar{\gamma}(0), t]}{\partial \bar{\gamma}_{m}(0) \partial \bar{\gamma}_{n}(0)} \\
& +O\left([\delta \gamma(0)]^{3}\right) \\
\equiv & A_{k l}+\sum_{m} \delta \gamma_{m}(0) B_{k l m} \\
& +\sum_{m n} \frac{1}{2} \delta \gamma_{m}(0) \delta \gamma_{n}(0) C_{k l m n}+O\left([\delta \gamma(0)]^{3}\right),
\end{aligned}
$$

where we have defined $A_{k l}, B_{k l m}$, and $C_{k l m n}$ as the zeroth-, first-, and second-order derivatives of the stability matrix at $\bar{\gamma}(0)$, respectively. The summation indices $m, n$, etc., run from 1 to 4 for a two-degree-of-freedom system, and $\delta \gamma(0) \equiv[\gamma(0)-\bar{\gamma}(0)]$. The time dependence of $A_{k l}, B_{k l m}$, and $C_{k l m n}$ is governed by the following set of first-order differential equations together with the canonical equations for classical trajectories:

$$
\begin{aligned}
& \frac{d A_{k l}}{d t}=\sum_{k^{\prime} l^{\prime}} J_{k l^{\prime}} \frac{\partial^{2} H}{\partial \gamma_{l^{\prime}} \partial \gamma_{k^{\prime}}} A_{k^{\prime} l}, \\
& \frac{d B_{k l m}}{d t}=\sum_{k^{\prime} l^{\prime}} J_{k l^{\prime}}\left(\sum_{n^{\prime}} \frac{\partial^{3} H}{\partial \gamma_{l^{\prime}} \partial \gamma_{k^{\prime}} \partial \gamma_{n^{\prime}}} A_{n^{\prime} m} A_{k^{\prime} l}\right. \\
& \left.+\frac{\partial^{2} H}{\partial \gamma_{l^{\prime}} \partial \gamma_{k^{\prime}}} B_{k^{\prime} l m}\right) \\
& \frac{d C_{k l m n}}{d t}=\sum_{k^{\prime} l^{\prime}} J_{k l^{\prime}}\left(\sum_{m^{\prime}} \frac{\partial^{3} H}{\partial \gamma_{l^{\prime}} \partial \gamma_{k^{\prime}} \partial \gamma_{m^{\prime}}} A_{m^{\prime}{ }_{n}} B_{k^{\prime} l m}\right. \\
& \left.+\frac{\partial^{2} H}{\partial \gamma_{l^{\prime}} \partial \gamma_{k^{\prime}}} C_{k^{\prime} l m n}\right)+\sum_{k^{\prime} l^{\prime} n^{\prime}} J_{k l^{\prime}} \frac{\partial^{3} H}{\partial \gamma_{l^{\prime}} \partial \gamma_{k^{\prime}} \partial \gamma_{n^{\prime}}} \\
& \times\left(B_{k^{\prime} l n} A_{n^{\prime} m}+B_{n^{\prime} m n} A_{k^{\prime} l}\right)+\sum_{k^{\prime} l^{\prime} n^{\prime} m^{\prime}} J_{k l^{\prime}}
\end{aligned}
$$

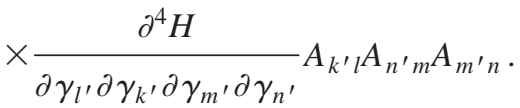

Consider two initial points in the phase space, $\gamma(0)$ and $\bar{\gamma}(0)$. They generate two classical trajectories, denoted by $\gamma(t)$ and $\bar{\gamma}(t)$, respectively. From the trajectory viewpoint, of most interest is the difference $\delta \gamma_{j}(t) \equiv\left[\gamma_{j}(t)\right.$ $\left.-\bar{\gamma}_{j}(t)\right]$, which is a differentiable function of $\bar{\gamma}(0)$, $\delta \gamma(0)$, and $t$. Fixing $\bar{\gamma}(0)$, and thus $\bar{\gamma}(t)$, gives a reference trajectory. Consider now the Taylor-series expansion of $\delta \gamma_{j}(t)$ in terms of $\delta \gamma(0)$. Obviously $\delta \gamma_{j}(t)=0 \quad$ if $\quad \delta \gamma(0)=0, \quad \partial \delta \gamma_{j}(t) /\left.\partial \delta \gamma_{l}(0)\right|_{\delta \gamma(0)=0}=A_{j l}$, $\partial^{2} \delta \gamma_{j}(t) /\left.\partial \delta \gamma_{k}(0) \partial \delta \gamma_{l}(0)\right|_{\delta \gamma(0)=0}=B_{j k l}$, and so on. In fact, the $n$ th-order derivative of $\delta \gamma_{j}(t)$ with respect to $\delta \gamma(0)$ is simply given by the $(n-1)$ th-order derivative of the stability matrix $\mathbf{M}[\bar{\gamma}(0), t]$ with respect to $\bar{\gamma}(0)$. Based on this observation, one obtains the following relation:

$$
\begin{aligned}
\sum_{l} A_{j l} \delta \gamma_{l}(0)= & \delta \gamma_{j}(t)-\frac{1}{2} \sum_{l k} \delta \gamma_{k}(0) \delta \gamma_{l}(0) B_{j k l} \\
& -\frac{1}{6} \sum_{j k l m} \delta \gamma_{k}(0) \delta \gamma_{l}(0) \delta \gamma_{m}(0) C_{j k l m} \\
& -O\left([\delta \gamma(0)]^{4}\right)
\end{aligned}
$$


Consider now the initial classical distribution function corresponding to the coherent state

$$
\begin{aligned}
\rho_{0}[\gamma(0), \bar{\gamma}(0)]= & \left(\frac{1}{4 \pi^{2} \sigma_{q_{1}} \sigma_{q_{2}} \sigma_{p_{1}} \sigma_{p_{2}}}\right) \exp \left[-\frac{\left(q_{1}-\bar{q}_{1}\right)^{2}}{2 \sigma_{q_{1}}^{2}}\right. \\
& \left.-\frac{\left(q_{2}-\bar{q}_{2}^{2}\right)}{2 \sigma_{q_{2}}^{2}}-\frac{\left(p_{1}-\bar{p}_{1}\right)^{2}}{2 \sigma_{p_{1}}^{2}}-\frac{\left(p_{2}-\bar{p}_{2}\right)^{2}}{2 \sigma_{p_{2}}^{2}}\right] .
\end{aligned}
$$

After lengthy calculations (see the Appendix) using Eqs. (9), (32), (34), and (35), we obtain an enlightening expression for $\chi_{2 c}^{2}(t)$,

$$
\begin{aligned}
\chi_{2 c}^{2}(t)= & \frac{2}{\hbar^{2}} \sum_{j}\left[\left\langle\left[\gamma_{j}(t)\right]^{2}\right\rangle_{0}-\left\langle\gamma_{j}(t)\right\rangle_{0}^{2}\right] \\
& -\frac{2}{\hbar^{2}} \sum_{j k k^{\prime} l l^{\prime}}\left(\frac{1}{4} B_{j k l^{\prime}} B_{j k^{\prime} l^{\prime}}+\frac{1}{3} A_{j k} C_{j k^{\prime} l l^{\prime}}\right) \\
& \times\left\langle\delta \gamma_{k}(0) \delta \gamma_{k^{\prime}}(0) \delta \gamma_{l}(0) \delta \gamma_{l^{\prime}}(0)\right\rangle_{0} \\
& +\sum_{j k k^{\prime} l l^{\prime}}\left(\frac{1}{4} B_{j k l^{\prime}} B_{j k^{\prime} l^{\prime}}+\frac{1}{4} A_{j k} C_{j k^{\prime} l l^{\prime}}\right) \\
& \times\left\langle\alpha_{k} \alpha_{k^{\prime}} \delta \gamma_{l}(0) \delta \gamma_{l^{\prime}}(0)\right\rangle_{0}+\frac{1}{\hbar^{2}} O\left([\delta \gamma(0)]^{6}\right) .
\end{aligned}
$$

Here $\langle\cdots\rangle_{0}$ represents the average over the initial classical ensemble. Note that the factor $\hbar^{2}$ in the above equation is due to the fact that $\sigma_{q_{1}} \sigma_{p_{1}}=\sigma_{q_{2}} \sigma_{p_{2}}=\hbar / 2$ for any classical distribution function corresponding to a minimumuncertainty-product state.

Equation (36) provides a quantitative connection between the development of phase-space structure and the instability characteristics of classical trajectories. Further, it allows for a closer examination of when and how classical distribution functions begin to develop a structure that differs from quantal distribution functions. Consider then, for simplicity, a special case in which the initial coordinate variances are identical to the initial momentum variances, i.e., $\sigma_{q_{1}}=\sigma_{p_{1}}$ $=\sigma_{q_{2}}=\sigma_{p_{2}}$. One obtains

$$
\chi_{2 c}^{2}(t)=\frac{2}{\hbar^{2}} \sum_{j}\left[\left\langle\left[\gamma_{j}(t)\right]^{2}\right\rangle_{0}-\left\langle\gamma_{j}(t)\right\rangle_{0}^{2}\right]+f(t)+O(\hbar),
$$

where $f(t)$ is given by

$$
\begin{aligned}
f(t)= & -\sum_{j k k^{\prime} l l^{\prime}}\left(\frac{1}{2} B_{j k l} B_{j k^{\prime} l^{\prime}}+\frac{2}{3} A_{j k} C_{j k^{\prime} l l^{\prime}}\right) \\
& \times\left[\frac{3}{4} \delta_{k k^{\prime} l l^{\prime}}+\frac{1}{4}\left[\left(\delta_{k k^{\prime}} \delta_{l l^{\prime}}+\delta_{k l^{\prime}} \delta_{l k^{\prime}}\right)\left(1-\delta_{k l}\right)\right.\right. \\
& \left.\left.+\delta_{k l} \delta_{k^{\prime} l^{\prime}}\left(1-\delta_{k k^{\prime}}\right)\right]\right]+\sum_{j k k^{\prime} l l^{\prime} m m^{\prime}} J_{k m^{\prime}} J_{k^{\prime} m^{\prime}} \\
& \times\left(B_{j k l} B_{j k^{\prime} l^{\prime}}+A_{j k} C_{j k^{\prime} l l^{\prime}}\right)\left[\frac{3}{4} \delta_{m m^{\prime} l l^{\prime}}\right. \\
& +\frac{1}{4}\left[\left(\delta_{m m^{\prime}} \delta_{l l^{\prime}}+\delta_{m l^{\prime}} \delta_{l m^{\prime}}\right)\left(1-\delta_{m l}\right)\right. \\
& \left.\left.+\delta_{m l} \delta_{m^{\prime} l^{\prime}}\left(1-\delta_{m m^{\prime}}\right)\right]\right] .
\end{aligned}
$$

This expression affords perspectives into QCC. Recall first, as shown in the preceding section [see Eq. (20)], that for pure state dynamics the quantal measure $\chi_{2 q}^{2}(t)$ of phasespace structure can be expressed in terms of the sum of second-order moments. This is more or less a consequence of quantization: the smallest scale in coordinates is related to the largest momentum component of the wave function, and vice versa. Clearly, this is in general not the case for classical distribution functions. Nevertheless, Eqs. (36) and (37) indicate that, to the lowest order, $\chi_{2 c}^{2}(t)$ can still be related to the sum of second-order statistical moments. Specifically, as shown in Eq. (37), for an initially symmetric coherent state the first term contributing to $\chi_{2 c}^{2}(t)$ is given by the sum of second-order moments divided by $\hbar^{2} / 2$, which is exactly the same as the result in Eq. (20) for $\chi_{2 q}^{2}(t)$. In addition, $\chi_{2 c}^{2}(t)$ contains additional contributions absent in the quantum dynamics. For example, the leading-order correction term $f(t)$ is independent of $\hbar$. As shown by Eq. (38), this term is determined by the stability characteristics $A_{j k}, B_{j k m}$, and $C_{j k m n}$ associated with the trajectory starting from the centroid of the initial Gaussian distribution. These results show intriguing similarities and differences between $\chi_{2 c}(t)$ and $\chi_{2 q}(t)$.

As a simple example of Eq. (37), consider a quadratic Hamiltonian systems such as the harmonic oscillator or the inverted harmonic-oscillator system, where classical and quantum mechanics are expected to agree. Here the time evolution is a linear canonical transformation in phase space; hence the stability matrix elements $\mathbf{M}_{j k}[\bar{\gamma}(0), t]$ do not depend on $\bar{\gamma}(0)$ and $B_{j k l}, C_{j k l m}$, and all other higher-order derivatives of the stability matrix with respect to $\bar{\gamma}(0)$ are zero. Thus, in such linear systems, $\chi_{2 c}^{2}(t)$ is precisely given by the sum of some second-order moments divided by $\hbar^{2} / 2$, in perfect correspondence with $\chi_{2 q}^{2}(t)$ [see Eq. (20)].

For the case of chaotic systems, one can estimate that the stability matrix increases exponentially, i.e., $A_{k l} \sim \exp (\lambda t)$, with $\lambda$ being the average exponential increase rate up to time $t$. Likewise, one expects $B_{k l m} \sim \exp (\lambda t), C_{k l m n} \sim \exp (\lambda t)$, etc. Equation (38) then suggests that $f(t) \sim f_{0} \exp (2 \lambda t)$. By contrast, the first term on the right-hand side of Eq. (37) is determined by second-order moments and cannot increase for 

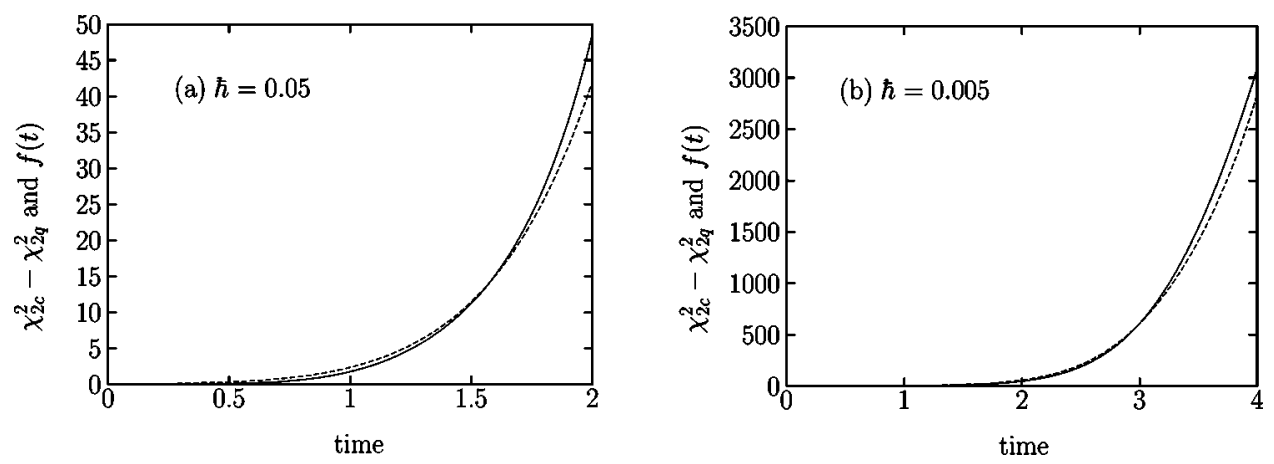

FIG. 4. Time dependence of $\left(\chi_{2 c}^{2}-\chi_{2 q}^{2}\right)$ compared with $f(t)$ in our analytical considerations [see Eqs. (37) and (38) in the text]. The initial condition is the minimum-uncertainty-product state considered in Sec. III. Dashed lines denote $\left(\chi_{2 c}^{2}-\chi_{2 q}^{2}\right)$ based on direct calculations in Sec. III and solid lines denote $f(t)$ obtained by directly integrating Eq. (38). $\hbar$ equals 0.05 in (a) and equals 0.005 in (b). The agreement between $\left(\chi_{2 c}^{2}-\chi_{2 q}^{2}\right)$ and $f(t)$ for the QCC break regime is excellent. All variables are in dimensionless units.

all time for bounded systems. Indeed, assuming that the characteristic magnitude of the second-order moments $\left[\left\langle\left(\gamma_{j}(t)\right)^{2}\right\rangle_{0}-\left\langle\gamma_{j}(t)\right\rangle_{0}^{2}\right](j=1,2,3,4)$ is given by $\Omega^{2}$, then the first term on the right-hand side of Eq. (37) would be bounded by $8 \Omega^{2} / \hbar^{2}$. Thus, in Eq. (37) the $f(t)$ term will be comparable to the preceding term after a time $t_{b}$, approximately given by

$$
t_{b}=\frac{1}{\lambda} \ln \left[\frac{\sqrt{8 \Omega^{2} / f_{0}}}{\hbar}\right] \text {. }
$$

A comparison between Eqs. (20) and (37) suggests that $t_{b}$ corresponds to the time scale after which classical descriptions of phase-space structure no longer agree with quantum results. Thus, $t_{b}$ can be identified as a logarithmic break time of QCC. This result is consistent with previous studies on the QCC break time using different approaches [24]. Note that, since Eq. (39) involves classical variables only, one can calculate $t_{b}$ without the need for any quantum calculations.

The origin of the classical-quantum difference lies in the $f(t)$ term in Eq. (37). This term does not have a quantal analog since it reflects classical phase-space structure that is beyond the resolution limit of quantal distribution functions [25]. To demonstrate the role of $f(t)$ we compare $\left[\chi_{2 c}^{2}(t)\right.$ $\left.-\chi_{2 q}^{2}(t)\right]$ from a direct calculation to $f(t)$ given by Eq. (38). Specifically, $f(t)$ is obtained from Eq. (38) by numerically computing the instability characteristics $A_{j k}, B_{j k l}$ and $C_{j k l m}$ via Eq. (33). Two cases with differing values of $\hbar$ have been examined. Results are shown in Figs. 4(a) and 4(b) that display the comparison between $\left[\chi_{2 c}^{2}(t)-\chi_{2 q}^{2}(t)\right]$ and $f(t)$ for both cases. The agreement is excellent, confirming the role of $f(t)$ in determining the break regime of QCC.

Figure 4 also shows some tiny discrepancies between $\left[\chi_{2 c}^{2}(t)-\chi_{2 q}^{2}(t)\right]$ and $f(t)$ (especially at later times), corresponding to higher-order contributions in Eq. (37). Nevertheless, the physics is still the same; i.e., the sum of $f(t)$ and these higher-order terms measures very fine phase-space structure that is unresolvable by the quantum distribution function.

\section{LONG AFTER THE BREAK REGIME}

The logarithmic break time $t_{b}$ of QCC introduces many issues regarding the relationship between quantum dynamics and classical mechanics. For instance, Zurek and Paz [26] showed that a logarithmic break time (somewhat different from that obtained above) can be counterintuitively short even for macroscopic objects. This being the case, our everyday experience in a macroscopic classical world full of chaotic events seems incompatible with the notion that classical physics is a large-quantum-number limit of quantum mechanics. Likewise, the smallness of the QCC break time seems to imply that classical physics cannot play a role in nonlinear molecular dynamics, contradicting the fact that classical physics often works well in many dynamics simulations. To at least partially resolve these puzzles, this section attempts to explore the implications of the quantum-classical discrepancy in phase-space structure for ensemble statistics.

As already implied by a comparison between Eqs. (20) and (37), what is directly responsible for the logarithmic break time is not the quantum-classical difference in expectation values, but simply the exponential increase of $f(t)$, which reflects the richness of fragmentation of classical distribution functions. As such, it is interesting to examine QCC in terms of some observables. Figure 5 displays the time dependence of four variances, i.e., $\left\langle q_{1}^{2}\right\rangle-\left\langle q_{1}\right\rangle^{2},\left\langle q_{2}^{2}\right\rangle$ $-\left\langle q_{2}\right\rangle^{2},\left\langle p_{1}^{2}\right\rangle-\left\langle p_{1}\right\rangle^{2}$, and $\left\langle p_{2}^{2}\right\rangle-\left\langle p_{2}\right\rangle^{2}$, for both classical and quantum dynamics. The initial state corresponds to that used in Figs. 3(a) and 4(b), and $\hbar$ still equals 0.005 , allowing direct comparison with the previous results.

Interestingly, results in Fig. 5 show that QCC in these second-order moments is excellent for times up to $t \approx 12.0$, which is much longer than the break time $t_{b} \sim 3.0$ identified in Fig. 3. In particular, for times less than $t=2.5$, both quantal and classical variances are seen to increase very rapidly, in exactly the same manner. This rapid increase is exponential in nature because, in this regime, the sum of these variances (divided by $\hbar^{2} / 2$ ) give $\chi_{2 c}^{2}$ (or $\chi_{2 q}^{2}$ ), which indeed increases exponentially on the average [see Fig. 3(a)]. This confirms a published suggestion that the initial rapid increase of both quantal and classical variances could be used to identify classical chaos from quantum dynamics [7]. For times 

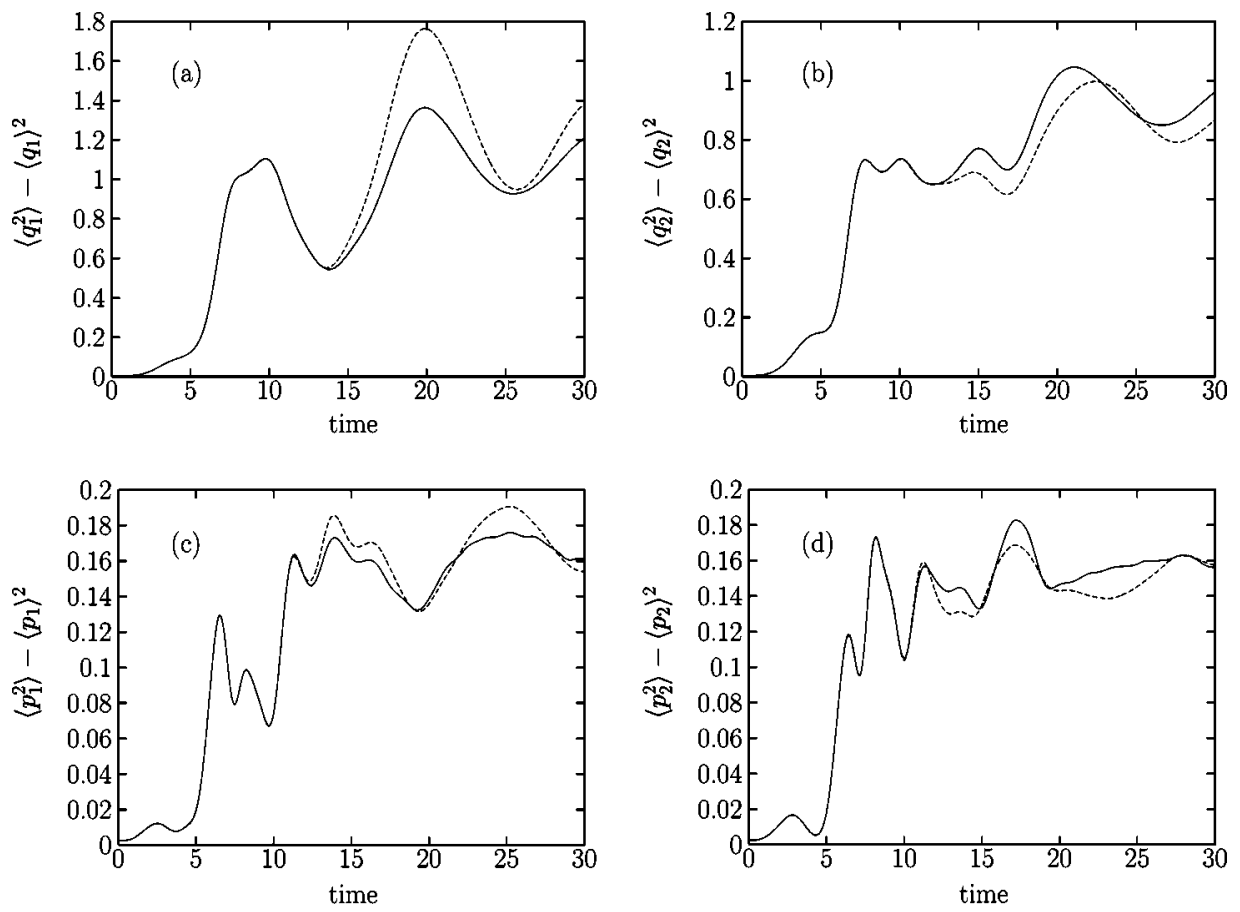

FIG. 5. Quantum-classical comparison of the time dependence of four second-order statistical moments, i.e., $\left\langle q_{1}^{2}\right\rangle-\left\langle q_{1}\right\rangle^{2}$ in (a), $\left\langle q_{2}^{2}\right\rangle-\left\langle q_{2}\right\rangle^{2}$ in (b), $\left\langle p_{1}^{2}\right\rangle$ $-\left\langle p_{1}\right\rangle^{2}$ in (c), and $\left\langle p_{2}^{2}\right\rangle-\left\langle p_{2}\right\rangle^{2}$ in (d), for times much larger than the logarithmic break time $t_{b}$. The initial distribution function corresponds to that in Figs. 3(a) and 4(b), and $\hbar=0.005$. Dashed lines denote classical results and solid lines denote quantum results. The QCC shown here during the complex relaxation process $(0<t$ $<12.0)$ is remarkable. All variables are in dimensionless units. $2.5<t<12.0$ during which excellent QCC persists, the second-order moments display a complex evolution. For example, there is clearly a diffusive regime between $t=5.0$ and $t=7.0$ with very large diffusion constants. After that, several very strong oscillations [particularly in Figs. 5(b)-(d)] can be observed, suggesting that both the quantal and classical distribution functions alternate between a high degree of delocalization and a certain degree of localization over the entire accessible phase space (this oscillatory behavior also suggests that the QCC time scale here should scale as a power of $\hbar$, rather than scale as $\ln \hbar$ ). The impressive QCC in this complex relaxation pattern is in sharp contrast to the results in Figs. 3(a) and 4(b), where quantum-classical differences, as quantitatively described by $\left[\chi_{2 c}^{2}(t)-\chi_{2 q}^{2}(t)\right]$ and predicted by $f(t)$, are already very large for $3.5<t$ $<4$.0. For even later times $(t>12.0)$, Fig. 5 shows that quantum-classical discrepancies begin to show up, but still with very similar trends in the oscillations.

These results indicate that the logarithmic break time may be quite irrelevant to QCC when viewed from the perspective of some low-order statistical moments [28]. This is the case since considering low-order statistical moments is equivalent to projecting the quantal and classical distribution functions onto a much smaller subspace. In this projection, or coarse-graining procedure, all the information encoded in an infinite number of higher-order statistical moments is lost. An accurate QCC is thus restored due to the loss of detailed information.

The results here also give further support to the idea of applying classical propagation methods to quantum distribution functions, as a means of approximating the true quantum mechanics [27]. That is, chaos may not cause a rapid failure of classical dynamics simulations if one is only interested in expectation values of some observables. More importantly, the results provide more insights into a fundamental issue regarding the role of decoherence in QCC [29,30]. On one hand, our results here show that during a relaxation process whose time scale is much larger than the logarithmic break time, decoherence may still be unnecessary in order to ensure QCC in low-order statistical moments. Thus, at least in our model system, decoherence effects on correspondence in expectation values of low-order statistical moments, if any, should be considered after the relaxation process is essentially completed, e.g., after $t=12.0$ in Fig. 5. This point supports the argument of Casati and Chirikov [30], extending their considerations from one-dimensional kicked systems to conservative systems, and agrees with the recent work [9] by Emerson and Ballentine. On the other hand, as shown in our previous work using the same model system [19], QCC for much larger time scales can be much improved with the introduction of decoherence.

\section{SUMMARY}

We have studied the issue of QCC in conservative chaotic systems in detail using a phase-space distribution function approach. The nature of QCC in three different regimes is exposed. In particular (i) the short-time increase rate of phase-space structure is studied in connection with finitetime Lyapunov exponents; (ii) a simple and enlightening description of the break regime of QCC is obtained by successfully accounting for the classical phase-space structure, which is beyond the quantum description; and (iii) excellent QCC in low-order statistical moments is shown to persist during a complex relaxation process, whose time scale is much larger than the logarithmic break time.

\section{ACKNOWLEDGMENTS}

This work was supported by the Natural Sciences and Engineering Research Council of Canada. We thank Professor Arjendu Pattanayak for useful discussions. 
APPENDIX: DERIVATION OF EQ. (36)

In this appendix we give a rather detailed derivation of Eq. (36) using Eqs. (9), (32), (34), and (35). We begin with the derivation by substituting Eq. (32) and the initial distribution function $\rho_{0}[\gamma(0), \bar{\gamma}(0)]$ [see Eq. (35)] into Eq. (9), evaluating the first derivatives of $\rho_{0}[\bar{\gamma}(0), \gamma(0)]$, and finally rescaling all integration variables by a factor of $1 / \sqrt{2}$. One then obtains

$$
\begin{aligned}
\chi_{2 c}^{2}(t)= & \frac{1}{2} \int d \gamma(0) \rho_{0}[\gamma(0), \bar{\gamma}(0)] \\
& \times \sum_{j} \mid \sum_{k} \alpha_{k}\left[A_{j k}+\sum_{m} \frac{1}{\sqrt{2}} B_{j k m} \delta \gamma_{m}(0)\right. \\
& \left.+\sum_{m n} \frac{1}{4} C_{j k m n} \delta \gamma_{m}(0) \delta \gamma_{n}(0)+O\left([\delta \gamma(0)]^{3}\right)\right]\left.\right|^{2},
\end{aligned}
$$

where

$$
\begin{gathered}
\alpha_{1}=\left[-\frac{p_{1}(0)-\bar{p}_{1}(0)}{\sigma_{p_{1}}^{2}}\right], \quad \alpha_{2}=\left[-\frac{p_{2}(0)-\bar{p}_{2}(0)}{\sigma_{p_{2}}^{2}}\right], \\
\alpha_{3}=\left[\frac{q_{1}(0)-\bar{q}_{1}(0)}{\sigma_{q_{1}}^{2}}\right], \quad \alpha_{4}=\left[\frac{q_{2}(0)-\bar{q}_{2}(0)}{\sigma_{q_{2}}^{2}}\right] .
\end{gathered}
$$

Since $A_{j k}, B_{j k m}$, and $C_{j k m n}$ do not depend upon $\gamma(0)$, and only the even functions of $\delta \gamma(0)$ will contribute when integrated over $\rho_{0}[\gamma(0), \bar{\gamma}(0)]$, Eq. (A1) can be further reduced to

$$
\begin{aligned}
\chi_{2 c}^{2}(t)= & \sum_{j k k^{\prime} m m^{\prime}}\left(\frac{1}{4} A_{j k} C_{j k^{\prime} m m^{\prime}}+\frac{1}{4} B_{j k m} B_{j k^{\prime} m^{\prime}}\right) \\
& \times\left\langle\alpha_{k} \alpha_{k^{\prime}} \delta \gamma_{m}(0) \delta \gamma_{m^{\prime}}(0)\right\rangle_{0} \\
& +\frac{1}{2} \int d \gamma \rho_{0}[\gamma(0), \bar{\gamma}(0])\left(\sum_{j}\left|\sum_{k} A_{j k} \alpha_{k}\right|^{2}\right) \\
& +O\left(\boldsymbol{\alpha}^{2}[\delta \gamma(0)]^{4}\right),
\end{aligned}
$$

where $\langle\cdots\rangle_{0}$ means the ensemble average over the initial Gaussian distribution.

To further simplify the expression for $\chi_{2 c}^{2}(t)$, we make a change of the integration variables in the second term on the right-hand side of Eq. (A3), i.e.,

$$
\begin{gathered}
-\left(p_{1}-\bar{p}_{1}\right) \rightarrow \frac{\sigma_{p_{1}}}{\sigma_{q_{1}}}\left(q-\bar{q}_{1}\right), \quad-\left(p_{2}-\bar{p}_{2}\right) \rightarrow \frac{\sigma_{p_{2}}}{\sigma_{q_{2}}}\left(q_{2}-\bar{q}_{2}\right), \\
\left(q_{1}-\bar{q}_{1}\right) \rightarrow \frac{\sigma_{q_{1}}}{\sigma_{p_{1}}}\left(p_{1}-\bar{p}_{1}\right), \quad\left(q_{2}-\bar{q}_{2}\right) \rightarrow \frac{\sigma_{q_{2}}}{\sigma_{p_{2}}}\left(p_{2}-\bar{p}_{2}\right) .
\end{gathered}
$$

Note that the Jacobi matrix of this transformation is a unity matrix and it has no effect on the form of $\rho_{0}[\gamma(0), \bar{\gamma}(0)]$. As a result of this coordinate transformation we have $\alpha_{k}$ $\rightarrow \delta \gamma_{k}(0) /\left(\sigma_{q_{1}} \sigma_{p_{1}}\right)=\delta \gamma_{k}(0) /\left(\sigma_{q_{2}} \sigma_{p_{2}}\right)$. This makes it possible to reexpress the second term on the right-hand side of Eq. (A3) by use of Eq. (34). Specifically,

$$
\begin{aligned}
\frac{1}{2} \int d & \gamma(0) \rho_{0}[\gamma(0), \bar{\gamma}(0)]\left(\sum_{j}\left|\sum_{k} A_{j k} \alpha_{k}\right|^{2}\right) \\
= & \frac{1}{2\left(\sigma_{q_{1}} \sigma_{p_{1}}\right)^{2}} \int d \gamma(0) \rho_{0}[\gamma(0), \bar{\gamma}(0)] \\
& \times\left(\sum_{j}\left|\sum_{k} A_{j k} \delta \gamma_{k}(0)\right|^{2}\right) \\
= & \frac{1}{2\left(\sigma_{q_{1}} \sigma_{p_{1}}\right)^{2}}\left[\sum_{j}\left(\left\langle\left[\gamma_{j}(t)\right]^{2}\right\rangle_{0}-\left\langle\gamma_{j}(t)\right\rangle_{0}^{2}\right)\right.
\end{aligned}
$$

$$
\begin{aligned}
& -\sum_{j k l} B_{j k l}\left\langle\delta \gamma_{j}(t) \delta \gamma_{k}(0) \delta \gamma_{l}(0)\right\rangle_{0} \\
& +\frac{1}{4} \sum_{j k l k^{\prime} l^{\prime}} B_{j k l} B_{j k^{\prime} l^{\prime}}\left\langle\delta \gamma_{k}(0) \delta \gamma_{k^{\prime}}(0) \delta \gamma_{l}(0)\right. \\
& \left.\times \delta \gamma_{l^{\prime}}(0)\right\rangle_{0}-\frac{1}{3} \sum_{j k l m} C_{j k l m}\left\langle\delta \gamma_{j}(t) \delta \gamma_{k}(0)\right. \\
& \left.\left.\times \delta \gamma_{l}(0) \delta \gamma_{m}(0)\right\rangle_{0}+O\left([\delta \gamma(0)]^{6}\right)\right]
\end{aligned}
$$

The second and the fourth terms in the above expression are a linear function of $\delta \gamma(t)$ and they can be further transformed into some functions of $\delta \gamma(0)$ by using Eq. (34) a second time. We then get

$$
\begin{aligned}
\frac{1}{2} \int d & \gamma(0) \rho_{0}[\gamma(0), \bar{\gamma}(0)]\left(\sum_{j}\left|\sum_{k} A_{j k} \alpha_{k}\right|^{2}\right) \\
= & \frac{1}{2\left(\sigma_{q_{1}} \sigma_{p_{1}}\right)^{2}} \sum_{j}\left[\left\langle\left(\gamma_{j}(t)\right)^{2}\right\rangle_{0}-\left\langle\gamma_{j}(t)\right\rangle_{0}^{2}\right] \\
& -\frac{1}{2\left(\sigma_{q_{1}} \sigma_{p_{1}}\right)^{2}} \sum_{j k k^{\prime} l l^{\prime}}\left(\frac{1}{4} B_{j k l} B_{j k^{\prime} l^{\prime}}+\frac{1}{3} A_{j k} C_{j k^{\prime} l l^{\prime}}\right) \\
& \times\left\langle\delta \gamma_{k}(0) \delta \gamma_{k^{\prime}}(0) \delta \gamma_{l}(0) \delta \gamma_{l^{\prime}}(0)\right\rangle_{0} \\
& +\frac{1}{\left(\sigma_{q_{1}} \sigma_{p_{1}}\right)^{2}} O\left([\delta \gamma(0)]^{6}\right)
\end{aligned}
$$

Finally, inserting Eq. (A6) into Eq. (A3) yields Eq. (36). 
[1] See, however, A.J. Pattanyak and P. Brumer, Phys. Rev. Lett. 77, 59 (1996), where exponential divergence in quantum wave packets is demonstrated.

[2] F. Haake, Quantum Signatures of Chaos (Springer-Verlag, New York, 1992).

[3] L.E. Reichl, The Transition to Chaos in Conservative Classical Systems: Quantum Manifestations (Springer-Verlag, New York, 1992).

[4] J. von Neumann, Mathematical Foundations of Quantum Mechanics (Princeton University Press, Princeton, NJ, 1955).

[5] B.O. Koopman, Proc. Natl. Acad. Sci. U.S.A. 17, 315 (1931).

[6] J. Wilkie and P. Brumer, Phys. Rev. A 55, 27 (1997); 55, 43 (1997).

[7] R.F. Fox and T.C. Elston, Phys. Rev. E 49, 3683 (1994).

[8] L.E. Ballentine, Y. Yang, and J.P. Zibin, Phys. Rev. A 50, 2854 (1994).

[9] J. Emerson and L.E. Ballentine, Phys. Rev. E 64, 026217 (2001); Phys. Rev. A 63, 052103 (2001).

[10] K.M. Christoffel and P. Brumer, Phys. Rev. A 33, 1309 (1985).

[11] A.K. Pattanayak and P. Brumer, Phys. Rev. E 56, 5174 (1997).

[12] Y. Gu, Phys. Lett. A 149, 95 (1990).

[13] M.H. Partovi, Phys. Rev. A 45, R555 (1992).

[14] A.K. Pattanayak and P. Brumer, Phys. Rev. Lett. 79, 4131 (1997).

[15] A.K. Pattanayak, Phys. Rev. Lett. 83, 4526 (1999).

[16] Note that if the system-environment coupling is nonlinear, then the decoherence rate and classical entropy production rate can be very different. For details see J. Gong and P. Brumer, Phys. Rev. Lett. 90, 050402 (2003).

[17] Z.P. Karkuszewski, C. Jarzynski, and W.H. Zurek, Phys. Rev. Lett. 89, 170405 (2002).

[18] B. Eckhardt, G. Hose, and E. Pollak, Phys. Rev. A 39, 3776 (1989).

[19] J. Gong and P. Brumer, Phys. Rev. E 60, 1643 (1999).

[20] R.L. Hudson, Rev. Mod. Phys. 6, 249 (1974).

[21] A. Isar, A. Sandulescu, and W. Scheid, Phys. Rev. E 60, 6371 (1999).

[22] V.V. Dodonov, E.V. Kurmyshe, and V.I. Manko, Phys. Lett. 79A, 150 (1980).

[23] M.D. Feit, J.A. Fleck, and A. Steiger, J. Comput. Phys. 47, 412 (1982).

[24] G.P. Berman and G.M. Zaslavsky, Physica A 91, 450 (1978); M.V. Berry, N.L. Balasz, M. Tabor, and A. Voros, Ann. Phys. (N.Y.) 122, 26 (1979).

[25] The resolution limit of quantal distribution functions due to the finite value of $\hbar$ stills allows for sub-Planck structures. For details see W.H. Zurek, Nature (London) 412, 712 (2001).

[26] W.H. Zurek and J.P. Paz, Phys. Rev. Lett. 72, 2508 (1994); Physica D 83, 300 (1995).

[27] C. Jaffe and P. Brumer, J. Phys. Chem. 88, 4829 (1984); J. Chem. Phys. 82, 2330 (1985).

[28] For a related conclusion, based on a comparison of phasespace densities, see Ref. [10].

[29] W.H. Zurek and J.P. Paz, Phys. Rev. Lett. 75, 351 (1995).

[30] G. Casati and B.V. Chirikov, Phys. Rev. Lett. 75, 350 (1995). 